\section{Looking back on 2019}

As Nature Catalysis starts its third year, we look back and the past 12 months.

$\mathrm{T}$ he end of 2019 marks another busy and successful year for Nature Catalysis. With the completion of volume 2, we have continued to publish some of the most exciting research in the various areas that comprise the broader field of catalysis.

One of the highlights for our editors was the Insight on catalysis for transport. This focused on three main areas where catalysis directly influences transportation: power supplies, emission abatement, and fuel synthesis. The mix of content - from industrial and academic researchers aligned well with our goal of bridging applied and fundamental sciences. It also featured one of our most-read pieces of the year: a Review Article on single-atom catalysis for automotive applications by Atsushi Beniya and Shougo Higashi at Toyota R\&D laboratories.

Overall, we slightly increased the amount of content we published. The breakdown in subject areas remained similar to the previous year, except for a significant increase in the amount of work on homogeneous catalysis (especially synthetic organic methodology). Again, the number of papers published in a given area tends to correlate well with the amount of submissions in that area - all subjects are given equal consideration when deciding what to publish.

In general our most downloaded content of the year was in electrocatalysis, with our most-read article in 2019 being a study on catalyst design for oxygen evolution in acidic electrolytes. On the other hand, when it comes to Altmetrics, it was the biological sciences that tended to get wider online attention. An Article reporting bioorthogonal catalysis by palladium did particularly well in this regard, followed by a Review Article providing a metabolic map for the production of bio-derived chemicals.

\section{"The breakdown in subject areas remained similar to the previous year, except for a significant increase in the amount of work on homogeneous catalysis (especially synthetic organic methodology)."}

Speaking of review content, we published a number of Review Articles, Perspectives and Comments that not only summarized research areas, but also added to them. Our most read Review Article of the year was on the design of catalysts and electrolytes for $\mathrm{CO}_{2}$ electroreduction. A Perspective in March proposed a rigorous protocol to try to ensure reproducible results in the electrochemical reduction of nitrogen to ammonia. The protocol stresses the importance of electrocatalytic tests and control experiments to quantify and avoid the presence of contaminants that would otherwise lead to false-positive results. Together with other recent publications nanosheets inside tumour-derived exosomes (for example, Nature 570, 504-508; 2019) this has contributed to setting a high standard for a very important and emerging topic.

And now with our first issue of 2020 we look forward to the coming year. There is some fantastic research in this issue: an air-stable source of nickel(0) promises to simplify the development of nickel-catalysed reactions. Sticking with organic synthetic methodology, we also have papers covering the asymmetric 1,1-difunctionalization of alkynes, photocatalytic activation of unreactive alkyl chlorides, and combined $\mathrm{N}$-heterocyclic carbene and iridium catalysis for the synthesis of $\gamma$-butyrolactones. In electrocatalysis, you can find work spanning $\mathrm{CO}_{2}$ reduction, hydrogen evolution, and ethylene glycol synthesis. From the biosciences side of things, we have work on the engineering of yeast to enhance the production of valuable fatty acids. And finishing things off, the issue also features two studies on heterogeneous systems: in situ monitoring of catalysed surface reactions by scanning electron microscopy and molten metal alloys for dry reforming of methane.

And finally, thanks again to the authors, reviewers and readers who continue to make Nature Catalysis such a success. We very much look forward to seeing what advances and insights 2020 will bring to the field of catalysis.

Published online: 22 January 2020 https://doi.org/10.1038/s41929-020-0424-2 